\title{
Atenção à saúde da criança na rede básica: análise de dissertações e teses de enfermagem
}

\section{Attention to child's health of primary care: analyze of dissertations and thesis of nursing}

\author{
Atención a salud del niño en la red básica de salud: análisis de disertación e \\ tesis de enfermería
}

\author{
Andresa Braun Novaczyk', Náudia da Silva Dias", Maria Aparecida Munhoz Gaíva'"I
}

\begin{abstract}
RESUMO
Este trabalho teve como objetivo analisar a produção científica dos programas de pósgraduação em enfermagem sobre a assistência à criança na atenção básica. A pesquisa documental foi realizada nos catálogos do Centro de Estudos e Pesquisas em Enfermagem (CEPEN), da Associação Brasileira de Enfermagem (ABEn), no período compreendido entre 2000 e 2006. Foram identificados 15 estudos que, após análise do conteúdo, permitiu a identificação de três categorias temáticas: a prática da enfermagem na atenção à saúde da criança na rede básica; a atenção à saúde da criança no PSF; a enfermagem e a busca pela assistência integral à saúde da criança. Concluise que a prática de enfermagem ainda está centrada no modelo médico hegemônico, o qual privilegia práticas prioritariamente curativas, fragmentadas e de baixa resolutividade. Porém, existem inúmeros esforços por parte dos enfermeiros na busca da promoção da saúde e da integralidade.
\end{abstract}

Palavras chave: Atenção Primária à saúde; Enfermagem Pediátrica; Cuidado da Criança.

\section{ABSTRACT}

This work had as objective to analyze the scientific production of masters degree course's programs in nursing about the assistance to children on health's basic area. The documental research was realized with the catalogs of the Center of Study and Research in Nursing (CEPEN), of the Nursing Brazilian Association (ABEn), in the period comprehended between 2000 and 2006. There were identified 15 studies that, after analysis of the content, helped to identify three thematic categories: the nurse's practice in the child's health on basic area; the attention to child's health in the Family Health Program (PSF); the nursing and the search for a whole assistance to children. The conclusion is that the practice of nursing is still focused in the hegemonic medical model, which privileges curative practices, which are fragmented and with a low resolution. However, there is a very good will to win this model and result in a promotion of health and integrally.

Key words: Primary Health Care; Pediatric Nursing; Child Care.

\section{RESUMEN}

Este trabajo es acerca de una pesquisa documental cuyo el objetivo es reflejar acerca de la participación de la enfermería en el atendimento básico de la salud del niño, tiendo como objeto de estudio la produción académica de la enfermería que hablas de la atención a la salud del niño en la red de salud. Este trabajo tuvo como objetivo analizar la produción de los programas de postgrado en enfermería sobre la asistencia al niño en atención básica. La pesquisa documental fue realizada en los catálogos del Centro de Estúdios y Pesquisas en Enfermería (CEPEN), de la Asociación Brasilera de Enfermería (ABEn), en el período comprendido entre 2000 y 2006. Fueran identificados 15 estudios que traen el análisis del contenido, que permitieron la identificación de tres categorias temáticas: la práctica de la enfermería en la atención a la salud del niño en la red básica; la atención a la salud del niño en el Programa de la Salud de la Família (PSF); la enfermería y la busca por asistencia integral para el niño. La conclusión es que la práctica de

Enfermeira. Mestranda do Curso de Enfermagem da Universidade Federal de Mato Grosso. Servidora Pública da Secretaria Estadual de Saúde do Mato Grosso. Cuiabá -MT. E-mail: andresabraun@hotmail.com.

"Enfermeira. Mestranda do Curso de Enfermagem da Universidade Federal de Mato Grosso. Docente da Faculdade de Enfermagem da Universidade de Cuiabá- UNIC e Universidade Estadual de Mato Grosso- UNEMAT. E-mail: naudia_dias@hotmail.com.

III Enfermeira. Professora Doutora do Departamento de Enfermagem e Coordenadora do Curso de Mestrado de Enfermagem da Universidade Federal de Mato Grosso. Email: mamgaiva@yahoo.com.br. 
Novaczyk AB, Dias NS, Gaíva MAM. Atenção à saúde da criança na rede básica: análise de dissertações e teses de enfermagem. Rev. Eletr. Enf. [Internet]. 2008;10(4):1124-37. Available from: http://www.fen.ufg. br/revista/v10/n4/v10n4a25.htm.

la enfermería aún está enfocada en el modelo médico hegemónico, o cual privilegia prácticas curativas, fragmentadas y con baja resolución. Sin embargo, existen diversos esfuerzos para vencer este modelo en la busca de la promoción

\section{NTRODUÇÃO}

A atenção à saúde da criança tem sido alvo de preocupação de profissionais da saúde, gestores e políticos, representando um campo prioritário de investimentos dentro dos cuidados à saúde da população.

Ao longo do tempo, percebe-se que em cada período histórico houve inúmeras transformações nas diretrizes das políticas de saúde voltadas à população infantil. Estas diretrizes enfocam um importante indicador, a mortalidade infantil, o qual reflete não apenas o nível de saúde, mas a qualidade de vida da população(1)

Mais recentemente, a taxa de mortalidade de menores de cinco anos vem sendo incorporada como um indicador fundamental do desenvolvimento humano e, por conseguinte, um importante indicador da situação da infância ${ }^{(1)}$

Os índices de mortalidade infantil no país, embora tenham reduzido na última década, ainda são altos, principalmente em algumas regiões como o Nordeste. As principais causas da mortalidade infantil estão ligadas diretamente às condições sócio-econômicas e culturais e dificuldades de acesso aos serviços de saúde. É contundente saber que a maioria destas mortes precoces pode ser evitada. De acordo com a Agenda de Compromissos para a Saúde Integral da Criança e Redução da Mortalidade Infantil, documento mais recente voltado à atenção a criança, elaborado pelo Ministério da Saúde em 2004, a promoção da saúde integral da criança e o desenvolvimento das ações de prevenção de agravos e assistência são objetivos que, para além da redução da mortalidade infantil, apontam para o compromisso de se prover qualidade de vida para a criança, ou seja, que esta possa crescer e desenvolver todo seu potencial ${ }^{(2)}$.

Diante deste cenário, muito tem sido feito nas últimas décadas com o objetivo de modificar as condições de saúde da população de la salud y de la integralidad.

Palabras clave: Atención Primaria de Salud; Enfermería Pediatrica; Cuidado del Niño.

infantil.

Em 1984, o Ministério da Saúde intensificou sua atuação na promoção da saúde de crianças até cinco anos de idade, através da criação do Programa de Assistência Integral à Saúde da Criança (PAISC). Este programa foi um grande marco assistencial, de uma política de universalização da assistência à saúde, objetivando a diminuição das condições que determinam a morbimortalidade infantil no país, nos últimos vinte anos ${ }^{(3)}$.

O PAISC, considerado uma política universal, porque visa o beneficio e alcance de toda população infantil brasileira, centra-se no desenvolvimento de cinco ações básicas de saúde integradas, capazes de responder aos problemas comuns da infância. São elas: Acompanhamento do Crescimento e Desenvolvimento Infantil; Programa de Incentivo ao Aleitamento Materno; Programa de Controle das Diarréias e Terapia de Reidratação Oral; e Programa de Assistência às Infecções Respiratórias Agudas ${ }^{(4)}$.

O objetivo do Ministério da Saúde quando apresentou esta proposta, era deslocar o enfoque de uma assistência médico-curativista, centrada na atenção à demanda espontânea às crianças doentes para garantir a integralidade na assistência prestada pelos serviços de saúde.

No entanto, embora as ações assistenciais à criança tenham uma filosofia de atenção integral, esta ainda está longe de ser alcançada, devido à fragilidade e fragmentação das ações oferecidas pelos profissionais da rede de serviços ${ }^{(3)}$.

Diante deste contexto e percebendo a necessidade de atividades mais amplas e integradas, três ações/estratégias foram incorporadas na última década à política de saúde do Brasil pelo Ministério da Saúde: o Programa Saúde da Família (PSF), a partir de 1994; a estratégia de Atenção Integrada às Doenças Prevalentes da Infância (AIDPI), em 1996; e a Agenda de Compromissos com a 
Novaczyk AB, Dias NS, Gaíva MAM. Atenção à saúde da criança na rede básica: análise de dissertações e teses de enfermagem. Rev. Eletr. Enf. [Internet]. 2008;10(4):1124-37. Available from: http://www.fen.ufg. br/revista/v10/n4/v10n4a25.htm.

Saúde Integral da Criança e Redução da Mortalidade Infantil, proposta em 2004 ${ }^{(2,5-6)}$. Estas ações objetivam um maior acesso aos cuidados básicos de saúde, tornando mais resolutivo o nível de atenção primária

Em termos de saúde infantil, fica evidente a evolução ocorrida na implementação de políticas assistenciais no país ao longo das últimas décadas do século passado. São ações de promoção do crescimento e desenvolvimento infantil saudáveis, enfocando a vigilância da saúde das crianças e o cuidado às doenças prevalentes, de modo articulado às diretrizes do PSF.

Considerando que a enfermagem é um grupo profissional com ampla responsabilidade na assistência à criança na atenção básica, interrogamos se esta nova forma de assistir através de ações de promoção da saúde infantil, englobando a abordagem coletiva e preconizando uma assistência integral, têm exercido em alguma medida, influências sobre a produção científica da enfermagem.

Diante do exposto, este trabalho tem como objetivo, analisar a produção científica dos programas de pós-graduação stricto sensu em enfermagem, voltada para a assistência à criança na atenção básica.

\section{METODOLOGI A}

Trata-se de uma pesquisa retrospectiva e documental, de abordagem qualitativa. Os estudos de natureza documental podem se constituir de uma abordagem valiosa sejam complementando as informações obtidas por outras técnicas, ou desvelando aspectos novos de um tema ou problema de estudo ${ }^{(7)}$.

O estudo foi realizado por meio de um levantamento dos catálogos do Centro de Estudos e Pesquisas em Enfermagem (CEPEN), da Associação Brasileira de Enfermagem (ABEn), identificando dissertações de mestrado e teses de doutorado que abordavam a atuação do enfermeiro na atenção à saúde da criança na rede básica de saúde. Optou-se por esta fonte de dados pela importância deste órgão na difusão de conhecimentos e por ele possuir o maior banco de teses e dissertações da área da enfermagem no Brasil. Além do mais, considera-se que nem toda produção da pós- graduação é divulgada em periódicos, dessa forma esse banco de dados foi considerado o mais apropriado para atingir 0 objetivo proposto.

Alguns critérios foram utilizados para a seleção do material:

- o resumo do trabalho deveria constar no catalogo do CEPEN, por ser este o órgão ligado à enfermagem e que tem $o$ compromisso de divulgar o conhecimento científico através do cadastramento de produções científicas de enfermeiros;

- os trabalhos deveriam ter sido realizados por enfermeiros e constar nas informações sobre pesquisas e pesquisadores em Enfermagem do CEPEN dos anos 2000 à 2006, limites de catalogação do CD-room disponíveis ao público, no momento de coleta dos dados desse estudo. Considera-se que esse período foi adequado para atingir o objetivo proposto.

- dissertações de mestrado e teses de doutorado, cujo objeto de estudo fosse a atenção à saúde da criança na rede básica.

Vale destacar que o recorte temporal foi definido em razão de que a última publicação dos catálogos do CEPEN é de 2006.

Após realizar a busca dos estudos, os mesmos foram solicitados, junto ao órgão mencionado. De posse do material foi realizada a leitura exploratória e analítica, para identificar o objeto, objetivos e os resultados do estudo; posteriormente, os dados foram registrados em um instrumento de coleta de dados denominado "Ficha de Análise". Nesta ficha constaram as referências bibliográficas das dissertações e teses, resumo do autor, resultados encontrados, contribuições para o papel da enfermagem na assistência à criança, entre outros dados. Os trabalhos foram identificados por "D" quando fizeram referência à dissertações, com suas respectivas numerações e por " $T$ " quando mencionadas as teses de doutorado. Em seguida efetivou-se a análise e interpretação do material, baseado na análise de conteúdo proposto por Gomes ${ }^{(8)}$ que aponta as seguintes etapas para operacionalização da técnica: recorte e classificação do material orientado 
Novaczyk AB, Dias NS, Gaíva MAM. Atenção à saúde da criança na rede básica: análise de dissertações e teses de enfermagem. Rev. Eletr. Enf. [Internet]. 2008;10(4):1124-37. Available from: http://www. fen.ufg. br/revista/v10/n4/v10n4a25.htm.

pelas questões de pesquisa; reconhecimento dos sentidos comuns e heterogêneos presentes por trás dos conteúdos manifestos; leitura transversal sobre os núcleos-categorias encontradas, reconhecendo-se os seus sentidos centrais; e articulação continua com a teoria. O conteúdo temático, encontrado nos trabalhos, foi categorizado segundo três núcleos temáticos: a prática da enfermagem na atenção à saúde da criança na rede básica; a atenção à saúde da criança no PSF; e a enfermagem e a busca pela assistência integral à saúde da criança.

\section{A produção do conhecimento sobre a Atenção à Saúde da Criança na rede Básica de Pós-Graduação em Enfermagem}

O levantamento das informações sobre pesquisas e pesquisadores em Enfermagem do CEPEN dos anos 2000 à 2006, nos permitiu localizar quinze trabalhos que tiveram como objeto de estudo, a atenção à criança na rede básica de saúde. A descrição detalhada dos mesmos pode ser observada no Quadro 1.

Quadro 1: Relação das dissertações e teses sobre a temática da atenção básica à saúde da criança em programas de Pós-Graduação em Enfermagem, disponíveis nos

catálogos do CEPEN/ABEn, dos anos 2000 a 2006.

\begin{tabular}{|c|c|c|c|c|c|}
\hline AUTOR & TITULO & TI PO & $\begin{array}{c}\text { ANO } \\
\text { DEFESA }\end{array}$ & LOCAL & I NSTI TUI ÇAO \\
\hline $\begin{array}{l}\text { Aranda, } \\
\text { DAL. }\end{array}$ & $\begin{array}{l}\text { As dimensões do cuidado à } \\
\text { criança: um diálogo entre } \\
\text { famílias e equipe de saúde na } \\
\text { comunidade. }\end{array}$ & $\begin{array}{c}\text { Tese } \\
\text { Doutorado }\end{array}$ & 2003 & $\begin{array}{l}\text { Rio de } \\
\text { Janeiro }\end{array}$ & $\begin{array}{c}\text { Escola de } \\
\text { Enfermagem Anna } \\
\text { Nery - Universidade } \\
\text { Federal do Rio de } \\
\text { Janeiro } \\
\end{array}$ \\
\hline Bordin, TA. & $\begin{array}{l}\text { O cuidado que influencia o ser } \\
\text { em desenvolvimento: a } \\
\text { enfermagem, a criança e a } \\
\text { família na sala de vacinas. }\end{array}$ & $\begin{array}{l}\text { Dissertação } \\
\text { Mestrado }\end{array}$ & 2000 & Florianópolis & $\begin{array}{l}\text { Universidade Federal } \\
\text { de Santa Catarina }\end{array}$ \\
\hline $\begin{array}{l}\text { Camargos, } \\
\text { CCRL. }\end{array}$ & $\begin{array}{c}\text { Atenção à saúde da criança de } 5 \\
\text { a } 10 \text { anos pela equipe Saúde da } \\
\text { Família em Juiz de Fora, Belo } \\
\text { Horizonte. }\end{array}$ & $\begin{array}{l}\text { Dissertação } \\
\text { Mestrado }\end{array}$ & 2002 & $\begin{array}{c}\text { Belo } \\
\text { Horizonte }\end{array}$ & $\begin{array}{c}\text { Escola de } \\
\text { Enfermagem- } \\
\text { Universidade Federal } \\
\text { de Minas Gerais } \\
\end{array}$ \\
\hline $\begin{array}{l}\text { Figueiredo, } \\
\text { GLA. }\end{array}$ & $\begin{array}{c}\text { A enfermagem no } \\
\text { acompanhamento do } \\
\text { crescimento e desenvolvimento } \\
\text { infantil em Unidade Básica de } \\
\text { Saúde: fragmentos e } \\
\text { reconstruções. }\end{array}$ & $\begin{array}{l}\text { Dissertação } \\
\text { Mestrado }\end{array}$ & 2001 & $\begin{array}{l}\text { Ribeirão } \\
\text { Preto }\end{array}$ & $\begin{array}{c}\text { Escola de } \\
\text { Enfermagem de } \\
\text { Ribeirão Preto- } \\
\text { Universidade de São } \\
\text { Paulo }\end{array}$ \\
\hline Lins, ICP. & $\begin{array}{c}\text { Organização da Atenção à saúde } \\
\text { da Criança: Programa de Saúde } \\
\text { da Família no Município de } \\
\text { Ilhéus- Ba. }\end{array}$ & $\begin{array}{l}\text { Dissertação } \\
\text { Mestrado }\end{array}$ & 2002 & Salvador & $\begin{array}{c}\text { Escola de } \\
\text { Enfermagem- } \\
\text { Universidade Federal } \\
\text { da Bahia }\end{array}$ \\
\hline $\begin{array}{l}\text { Lopes, } \\
\text { MSV. }\end{array}$ & $\begin{array}{l}\text { Cuidando da criança desnutrida } \\
\text { no contexto de sobrevivência e } \\
\text { resistência. }\end{array}$ & $\begin{array}{l}\text { Dissertação } \\
\text { Mestrado }\end{array}$ & 2001 & Fortaleza & $\begin{array}{c}\text { Universidade Federal } \\
\text { do Ceará }\end{array}$ \\
\hline $\begin{array}{l}\text { Martins, } \\
\text { DC. }\end{array}$ & $\begin{array}{l}\text { Seguimento de enfermagem às } \\
\text { crianças menores de um ano de } \\
\text { idade em uma unidade de saúde } \\
\text { da família de Ribeirão Preto-SP. }\end{array}$ & $\begin{array}{l}\text { Dissertação } \\
\text { Mestrado }\end{array}$ & 003 & $\begin{array}{l}\text { Ribeirão } \\
\text { Preto }\end{array}$ & $\begin{array}{c}\text { Escola de } \\
\text { Enfermagem de } \\
\text { Ribeirão Preto- } \\
\text { Universidade de São } \\
\text { Paulo } \\
\end{array}$ \\
\hline $\begin{array}{l}\text { Monteiro, } \\
\text { Al. }\end{array}$ & $\begin{array}{l}\text { As representações sociais da } \\
\text { prática de enfermagem no } \\
\text { interior dos programas de } \\
\text { atenção à saúde da criança de } \\
\text { uma unidade básica de saúde } \\
\text { do município de Natal. }\end{array}$ & $\begin{array}{c}\text { Tese } \\
\text { Doutorado }\end{array}$ & 2000 & $\begin{array}{l}\text { Ribeirão } \\
\text { Preto }\end{array}$ & $\begin{array}{c}\text { Escola de } \\
\text { Enfermagem de } \\
\text { Ribeirão Preto- } \\
\text { Universidade de São } \\
\text { Paulo }\end{array}$ \\
\hline Perillo, RD. & $\begin{array}{l}\text { Vigilância à Saúde das Crianças } \\
\text { menores de um ano no Centro } \\
\text { de Saúde Goiânia, Belo }\end{array}$ & $\begin{array}{l}\text { Dissertação } \\
\text { Mestrado }\end{array}$ & 2001 & $\begin{array}{l}\text { Belo } \\
\text { Horizonte }\end{array}$ & $\begin{array}{c}\text { Escola de } \\
\text { Enfermagem- } \\
\text { Universidade Federal }\end{array}$ \\
\hline
\end{tabular}


Novaczyk AB, Dias NS, Gaíva MAM. Atenção à saúde da criança na rede básica: análise de dissertações e teses de enfermagem. Rev. Eletr. Enf. [Internet]. 2008;10(4):1124-37. Available from: http://www.fen.ufg. br/revista/v10/n4/v10n4a25.htm.

\begin{tabular}{|c|c|c|c|c|c|}
\hline & Horizonte: limites e desafios. & & & & de Minas Gerais \\
\hline $\begin{array}{l}\text { Prado, } \\
\text { SRLA. }\end{array}$ & $\begin{array}{l}\text { Integralidade: um estudo a } \\
\text { partir da atenção básica à saúde } \\
\text { da criança em modelos } \\
\text { assistenciais distintos. }\end{array}$ & $\begin{array}{c}\text { Tese } \\
\text { Doutorado }\end{array}$ & 2005 & São Paulo & $\begin{array}{c}\text { Escola de } \\
\text { Enfermagem- } \\
\text { Universidade de São } \\
\text { Paulo }\end{array}$ \\
\hline Rocha, SS. & $\begin{array}{l}\text { Enfermeiros da estratégia saúde } \\
\text { da família no cuidado à saúde } \\
\text { das crianças em Teresina. }\end{array}$ & $\begin{array}{c}\text { Tese } \\
\text { Doutorado }\end{array}$ & 2005 & $\begin{array}{l}\text { Rio de } \\
\text { Janeiro }\end{array}$ & $\begin{array}{c}\text { Escola de } \\
\text { Enfermagem Anna } \\
\text { Nery - Universidade } \\
\text { Federal do Rio de } \\
\text { Janeiro } \\
\end{array}$ \\
\hline Santos, CC & $\begin{array}{c}\text { Avaliação da implementação da } \\
\text { estratégia de Atenção Integrada } \\
\text { às Doenças Prevalentes na } \\
\text { Infância (AIDPI) no Estado da } \\
\text { Bahia. }\end{array}$ & $\begin{array}{c}\text { Dissertação } \\
\text { Mestrado }\end{array}$ & 2004 & Salvador & $\begin{array}{c}\text { Escola de } \\
\text { Enfermagem- } \\
\text { Universidade Federal } \\
\text { da Bahia }\end{array}$ \\
\hline Silva, MEO. & $\begin{array}{c}\text { A prática da enfermeira a } \\
\text { assistência à saúde da criança } \\
\text { de } 0 \text { a } 1 \text { ano, na rede básica do } \\
\text { distrito sanitário Nordeste, } \\
\text { município de Belo Horizonte. }\end{array}$ & $\begin{array}{c}\text { Dissertação } \\
\text { Mestrado }\end{array}$ & 2003 & $\begin{array}{c}\text { Belo } \\
\text { Horizonte }\end{array}$ & $\begin{array}{c}\text { Escola de } \\
\text { Enfermagem- } \\
\text { Universidade Federal } \\
\text { de Minas Gerais }\end{array}$ \\
\hline $\begin{array}{c}\text { Siqueira, } \\
\text { RC. }\end{array}$ & $\begin{array}{l}\text { Diagnósticos de enfermagem } \\
\text { em crianças menores de um ano } \\
\text { acompanhadas na consulta de } \\
\text { enfermagem em puericultura } \\
\text { numa unidade básica de saúde } \\
\text { de Fortaleza-CE. }\end{array}$ & $\begin{array}{c}\text { Dissertação } \\
\text { Mestrado }\end{array}$ & 2005 & Fortaleza & $\begin{array}{c}\text { Faculdade de } \\
\text { Farmácia, } \\
\text { Odontologia e } \\
\text { Enfermagem- } \\
\text { Universidade Federal } \\
\text { do Ceará } \\
\end{array}$ \\
\hline Slomp, FM. & $\begin{array}{c}\text { Assistência ao recém-nascido } \\
\text { em uma unidade do Programa } \\
\text { de Saúde da Família de } \\
\text { Guarapuava-PR. }\end{array}$ & $\begin{array}{c}\text { Dissertação } \\
\text { Mestrado }\end{array}$ & 2005 & $\begin{array}{c}\text { Ribeirão } \\
\text { Preto }\end{array}$ & $\begin{array}{c}\text { Escola de } \\
\text { Enfermagem de } \\
\text { Ribeirão Preto- } \\
\text { Universidade de São } \\
\text { Paulo } \\
\end{array}$ \\
\hline
\end{tabular}

Como é possível notar, do conjunto de estudos analisados, quatro eram teses de doutorado e onze dissertações de mestrado, sendo observada uma maior predominância da produção nos Programas de Pós-Graduação da região Sudeste. Destes, três foram produzidos na Universidade Federal de Minas Gerais, cinco na Escola de Enfermagem de Ribeirão PretoUSP. Cabe destacar que esses achados são decorrentes do fato de a maioria dos cursos de pós-graduação da enfermagem estar localizados na região sudeste. Nos últimos anos, a política de pós-graduação brasileira tem incentivado a criação de cursos nas regiões centro-oeste e norte, para ampliação e fortalecimento da qualidade de ensino, produção do conhecimento científico e desenvolvimento de inovação tecnológica em regiões mais carentes do país. 0 maior número de trabalhos foi produzido no ano de 2005, com duas dissertações e duas teses, seguido de três produzidos em 2001 e 2003, dois em 2002 e 2000 e um trabalho realizado no ano de 2004.

Em relação à natureza das dissertações/teses, predominou os estudos qualitativos (10), em detrimento dos quantitativos (05). A pesquisa qualitativa de acordo com a autora ${ }^{(9)}$, responde a questões muito particulares, ou seja, trabalha com um universo de significados, motivações, aspirações, crenças, valores e atitudes, o que corresponde a um espaço mais profundo das relações, dos processos e dos fenômenos. Essa identificação da enfermagem com a abordagem qualitativa é justificada por seu objeto de cuidado ser o "homem", e para tal, necessita conhecer suas percepções e subjetividades para executar o cuidado.

Os referenciais teórico-metodológicos que ofereceram sustentação às discussões realizadas pelos diversos autores foi predominantemente 0 materialismo histórico/dialético, com cinco trabalhos. Foram utilizados ainda a hermenêutica, a fenomenologia, a etnografia, o método sensível e criativo, epidemiológico, teoria humanista e teoria das relações sociais.

$$
\text { A opção pelo materialismo }
$$

histórico/dialético se dá pela sua adequação à análise dos fenômenos sociais e à observação 
Novaczyk AB, Dias NS, Gaíva MAM. Atenção à saúde da criança na rede básica: análise de dissertações e teses de enfermagem. Rev. Eletr. Enf. [Internet]. 2008;10(4):1124-37. Available from: http://www.fen.ufg. br/revista/v10/n4/v10n4a25.htm.

da realidade em que a contradição e o conflito predominam sobre a harmonia e o consenso. A autora ${ }^{(9)}$ considera que "o materialismo histórico representa o caminho teórico que aponta a dinâmica do real na sociedade, a dialética refere-se ao método de abordagem deste real. Esforça-se para entender o processo histórico em seu dinamismo, provisoriedade e transformação. Busca apreender a prática social empírica dos indivíduos em sociedade, e realizar a crítica das ideologias (...)".

Apresentamos a seguir, a análise e discussão dos dados identificados a partir da leitura das teses e dissertações sobre a atenção à saúde da criança na rede básica.

\section{A prática da enfermeira na Atenção à Saúde da criança na Rede Básica}

Foi evidenciada nos trabalhos analisados uma preocupação em relação à organização do trabalho de enfermagem no atendimento à criança na rede básica. A atuação do enfermeiro na atenção básica tem se diversificado, pois além das atividades assistenciais, ele desenvolve atividades educativas e prioritariamente as administrativas.

A cada ano, no mundo, mais de onze milhões de crianças morrem ao contrair doenças infecciosas e em conseqüência de uma nutrição inadequada. Diante desta situação, faz-se necessário definir estratégias que proporcionem maior acesso aos cuidados básicos de saúde, focalizando a atenção nas populações de maior risco e na revitalização da atenção primária, tornando-a mais resolutiva e eficiente e possibilitando um atendimento de qualidade às crianças acometidas pelas doenças de maior prevalência na infância ${ }^{(10)}$.

Ao considerar os princípios da atenção primária à saúde, a Estratégia de Atenção Integrada às Doenças Prevalentes da Infância (AIDPI), vem se somar ao conjunto de ações específicas já disponíveis: PACS e PSF, promovendo uma maior coordenação e integração de atividades que possam contribuir para um manejo e prevenção das doenças mais adequadas e garantir a integralidade na assistência prestada pelos serviços de saúde. A AIDPI tem como objetivo diminuir a mortalidade em menores de cinco anos, causada pelas doenças prevalentes e que podem ser evitadas ou tratadas mediante a aplicação de normas de avaliação, classificação, tratamento, seguimento e orientações à família para o cuidado com a criança no domicílio.

$\mathrm{Na}$ reordenação do modelo da saúde, a enfermeira tem relevância no que diz respeito à implantação de estratégias, como a AIDPI. Estudo ${ }^{(10)}$ avaliando esta estratégia no estado da Bahia mostrou que o número de enfermeiras capacitadas em AIDPI é maior que o número de médicos. A autora ainda destaca que:

A enfermeira também exerce uma carga horária de trabalho que a faz estar presente por maior tempo nos serviços. Portanto, as enfermeiras estão mais envolvidas com as atividades, mais aptas e dispostas a seguir a metodologia da assistência, o que exige atenção e tempo para o atendimento e possuem historicamente preparo na atenção à criança na rede básica (D.2) .

A SESAB tem orientado os municípios na elaboração de protocolos de atendimento que legitimam a prescrição das enfermeiras para tratamentos padronizados dos programas de saúde pública, incluindo a AIDPI, definidos pelo Ministério da Saúde (D.2).

Este último trecho mostra a valorização do profissional enfermeiro na implementação de estratégias como a AIDPI. O cuidado da enfermeira, na atenção básica, é fundamental na promoção e prevenção da saúde e recuperação dos indivíduos. A enfermeira vem se destacando como um agente chave, inserida em um processo de trabalho complexo.

Outro estudo que caracteriza a demanda de crianças menores de um ano de idade cadastradas em uma Unidade Saúde da Família, também destaca a importância da enfermeira na implementação da estratégia AIDPI:

(...) destacamos a importância da criação de um instrumento para a sistematização da assistência à criança, como um guia para o atendimento e a implementação da estratégia da atenção integrada às doenças prevalentes na infância de forma mais efetiva e sistematizada para avançar rumo a atenção integral à saúde da criança e família (D.6).

A enfermagem compõe um grupo profissional responsável por diversas tarefas na atenção à criança na unidade básica de saúde 
Novaczyk AB, Dias NS, Gaíva MAM. Atenção à saúde da criança na rede básica: análise de dissertações e teses de enfermagem. Rev. Eletr. Enf. [Internet]. 2008;10(4):1124-37. Available from: http://www.fen.ufg.br/revista/v10/n4/v10n4a25.htm.

(UBS). Tarefas que vão além da realização de técnicas e da utilização de instrumentos, e sim de uma co-responsabilidade pela saúde da criança.

Identificamos nos trabalhos, que a enfermeira deve prestar o atendimento à criança de forma que favoreça o crescimento e o desenvolvimento saudáveis e de acordo com suas necessidades.

(...) a enfermeira deve estar organizada para o primeiro atendimento da criança na UBS, oferecendo um atendimento mais integral com ações educativas efetivas, garantindo a essa criança o vínculo com a unidade para o acompanhamento do seu crescimento e desenvolvimento (D.9).

É proposto que o bebê e família passem por consulta de enfermagem com enfoque em puericultura (...). Na consulta de enfermagem, realizada pela enfermeira, será observado o crescimento e desenvolvimento infantil, o aleitamento materno e as condições clínicas do bebê, assim como o agendamento de retorno (D.11).

As enfermeiras estão mais envolvidas com as atividades...., mais dispostas a seguir a metodologia da assistência...e preparo na atenção à criança na rede básica (D.2).

(...) a necessidade de a enfermagem estar ciente das condições sócio-econômico-culturais em que está inserida a criança, para prestar uma assistência de acordo com a população (D.3).

Neste contexto, cabe à enfermeira identificar o melhor cuidado à saúde da criança e família, pois no cotidiano da rede básica de saúde, no atendimento em pediatria, além da criança, ser biológico e social, insere-se a mãe, a família, suas subjetividades, compreensões, dúvidas e necessidades. É um encontro entre pessoas, de produção de relações, de diálogos e de atendimento às necessidades por parte daquele que busca algo ${ }^{(11)}$.

$\mathrm{Na}$ saúde coletiva, o trabalho de enfermagem tem se diversificado, indo desde o "cuidar" de enfermagem, seja do indivíduo, família e grupos da comunidade, passando pelas ações educativas, administrativas, até a participação no planejamento em saúde ${ }^{(12)}$.

Alguns autores dos estudos que compõem este trabalho evidenciam uma preocupação com o fato de a enfermeira estar priorizando as ações administrativas no processo de trabalho em UBS em detrimento das atividades assistenciais:

A demanda excessiva observada no trabalho da enfermagem, as rotinas das instituições, roubam o tempo que a enfermagem deveria ter para prestar o cuidado exclusivo à pessoa e poder refletir sobre sua própria prática e tornando-a mais humanizada (D. 7).

A enfermagem faz corretamente os procedimentos, procura atender bem a clientela, orienta, educa, organiza o serviço, mas possui uma rotina muito rígida e tarefeira (D.9).

(...) as normas rígidas, criadas nos serviços de saúde, assumidas pela enfermagem, podem colocar em risco o processo de cuidar de enfermagem (D.7).

Analisando as características do processo de cuidado da criança entendemos que a enfermeira deveria estar à frente das ações assistenciais e educativas, ou seja, ações de enfermagem propriamente ditas, ao invés de priorizar as atividades administrativas.

Diante dos novos paradigmas assistenciais, os enfermeiros, assim como os outros profissionais, enfrentam o desafio de rever sua prática. Considerando a complexidade que é atender a criança e família em seu contexto; assistir de forma integral e humanizada, resolutiva e com boa qualidade, pode gerar inseguranças, expectativas e frustrações. Neste sentido, a formação dos profissionais de saúde é uma questão que merece atenção se realmente quisermos mudar o modelo de atenção, pois ainda hoje essa formação se baseia numa visão cartesiana de divisão corpo e mente, desqualificando assim aspectos psicológicos, sociais e ambientais envolvidos no processo de cuidar.

Alguns autores ${ }^{(13)}$, assumem que a formação dos profissionais de saúde tem se mostrado alheia à necessidade de se promover uma educação baseada nos princípios e diretrizes do SUS, perpetuando assim o modelo hegemônico de formação, o qual necessita se estruturar com base na problematização do processo de trabalho e da sua capacidade de 
Novaczyk AB, Dias NS, Gaíva MAM. Atenção à saúde da criança na rede básica: análise de dissertações e teses de enfermagem. Rev. Eletr. Enf. [Internet]. 2008;10(4):1124-37. Available from: http://www.fen.ufg. br/revista/v10/n4/v10n4a25.htm.

atender adequadamente às necessidades de saúde das pessoas e das populações.

A prática de enfermagem inserida na atenção à saúde da criança na rede básica de saúde deveria priorizar as ações de prevenção e promoção de saúde. Estas ações devem ser compartilhadas pela equipe de profissionais da unidade básica de saúde, de maneira que cada um, dentro de seu espaço, desenvolva ações conjuntas em prol de uma melhor assistência à criança.

Porém o que vemos na prática ainda está longe de alcançar o ideal de atenção integral à saúde da criança. A enfermeira ainda está inserida em um contexto centrado no modelo biomédico hegemônico. Em um estudo realizado em Ribeirão Preto, foi demonstrado que

(...) as ações de enfermagem em Unidade Básica de Saúde são tomadas como meio do trabalho médico, pois auxilia e complementa a consulta médica colhendo os exames necessários, fazendo a pré e pós-consulta, decodificando as ordens médicas para os clientes ( D.4).

De modo geral, esse modelo ainda está vigente e se faz necessário buscar novos modelos de organização dos serviços que viabilizem oferecer adequadamente informações/orientações, cuidados, assistência acolhedora e humanizada e que possibilite à unidade de saúde tornar um espaço social, onde seja possível trabalhar questões individuais e coletivas $^{(11)}$.

Outro aspecto evidenciado nos estudos analisados é o enraizamento do modelo procedimento centrado na percepção da sociedade e sua influência para a prática da enfermagem.

A prática de enfermagem na atenção à saúde da criança, é influenciada, na maioria das vezes, pelo senso comum, dentro do modelo assistencial clínico individual e hegemônico (T.2).

O estudo das representações sociais da prática de enfermagem em unidades básicas de saúde $^{(14)}$ mostra que as mães das crianças desejam o atendimento curativo em detrimento do trabalho preventivo e educacional. Isto reflete o senso comum da mentalidade curativa conjugada com o modelo de saúde hegemônico.
Nesse modo de produção de saúde, a população valoriza a resolutividade dos problemas pelo qual procura a unidade básica de saúde através da demanda espontânea, inserida em um contexto de pronto atendimento. Para muitos vale mais uma receita de medicamentos do que mudar um hábito de vida, por exemplo. Dessa forma, a atuação da enfermagem no moldes do Pronto Atendimento é comprometida, limitada e restrita à atividade auxiliar da consulta médica(12). Apesar de predominar o modelo biomédico na atenção à criança, existem esforços para mudá-lo, que são evidenciados nos trechos a seguir:

(...) as ações de promoção da saúde ainda são desenvolvidas de modo tímido, sem grande ênfase, percebendo que a prática dos trabalhadores, entre eles a enfermagem, precisa estar mais pautada pelos princípios da promoção da saúde (D.5).

(...) as atividades de promoção e de atenção básica são fundamentais para a melhoria da qualidade de vida da população e as ações educativas são as principais estratégias em direção à promoção da saúde, (...) e exige um esforço contínuo dos profissionais de saúde no sentido de contribuir para o melhoramento das condições de saúde da população infantil (T.3).

Apesar da luta pela mudança do modelo assistencial, enfocado na promoção e prevenção da saúde, a enfermagem também luta para combater o modelo biomédico vigente (T.2).

A enfermagem deve refletir sobre como está sendo sua prática, definindo melhor o que é, como é, e para quem é o cuidado de enfermagem no processo de acompanhamento e desenvolvimento infantil na rede básica de saúde. É necessário que esta categoria profissional, repense sobre seu papel neste modelo biomédico, lutando por uma reversão de valores nas práticas profissionais vigentes, e enfoque seu cuidado no sentido de promover a saúde e buscar a atenção integral à saúde infantil.

\section{A atenção à saúde da criança no PSF}

Os trabalhos analisados trazem a importância da prática da enfermagem e do papel do enfermeiro na busca por um novo modelo de atenção. Dentre as estratégias de 
Novaczyk AB, Dias NS, Gaíva MAM. Atenção à saúde da criança na rede básica: análise de dissertações e teses de enfermagem. Rev. Eletr. Enf. [Internet]. 2008;10(4):1124-37. Available from: http://www.fen.ufg. br/revista/v10/n4/v10n4a25.htm.

mudança do modelo assistencial, o PSF tem sido - pilar na reestruturação do processo de trabalho das equipes de saúde. Inserir a assistência voltada para a criança neste contexto tem sido um grande desafio para essas equipes, tendo em vista a complexidade do processo saúde-doença da população infantil.

O Programa Saúde da Família, criado em 1994 pelo Ministério da Saúde surgiu como uma estratégia para reordenar o modelo de atenção à saúde e reorganizar a prática assistencial nas Unidades Básicas de Saúde ${ }^{(5)}$. É uma estratégia de caráter multiprofissional e que estabelece uma relação de vínculo com a comunidade, prestando um atendimento integral e priorizando a prevenção e promoção da saúde.

A enfermeira se integra as práticas de outros trabalhadores de saúde, compondo a equipe multiprofissional da USF que responde pela produção de serviços em saúde. Dessa forma, a prática da enfermeira é parte de um processo coletivo de trabalho que tem como finalidade, produzir ações de saúde, caracterizando-se por um saber específico, com ações contínuas e articuladas com os demais membros da equipe, na construção dos objetos comuns de trabalho no setor saúde ${ }^{(12) .}$

O trabalho da enfermeira é visto como consistente no movimento de construção das equipes nas unidades de saúde, buscando realizar a assistência integral.

A concretização do Sistema Único de Saúde propiciou inúmeras mudanças no modelo de atenção à saúde, e a enfermeira, neste contexto de transformações, deve incorporar novos conceitos aplicáveis ao processo de trabalho no setor saúde para promover a saúde da população infantil.

Os diversos estudos que compõem este trabalho mencionam que o enfermeiro, assim como toda a equipe de saúde da família, encontra barreiras para a efetivação dos propósitos do Programa Saúde da Família, principalmente no que se refere à assistência à saúde da criança.

(...) defronta-se hoje com um Sistema de Saúde restrito quanto a cobertura oferecida, de forma segmentada na produção e no acesso aos serviços. Dentre os que estão excluídos do acesso aos serviços de saúde ou 'fora dos programas' estão as crianças de 5 a 10 anos que sofrem com problemas que podem ser controlados com ações de promoção à saúde e de prevenção de agravos (D.3).

(...) o efeito sobre a situação de saúde-doença da população ainda não revela a incorporação do princípio da integralidade, sobretudo com relação ao caráter curativo da atenção prestada e da dificuldade de acesso (T.3).

Apesar das propostas de novas estratégias, as ações promocionais de saúde da criança pouco têm se expandido diferentemente do discurso oficial, e o que funciona é o modelo assistencial clínico individual, que prioriza o pronto-atendimento, e a medicalização. Talvez isto justifique a existência ainda de altas taxas de morbimortalidade infantil em algumas regiões causadas por doenças evitáveis.

Outra pesquisa analisada ${ }^{(15)}$ mostra que a Unidade Básica do Centro de Saúde Goiânia, em Belo Horizonte-MG, trabalha com informações territorializadas relativas aos nascimentos e aos óbitos, porém as ações não são orientadas pela vigilância à saúde como prática sanitária, pois o atendimento não privilegia as crianças consideradas de risco.

A discussão acerca da vigilância em saúde tem sido ampliada e hoje já se considera que possua as seguintes características: intervenção sobre problemas de saúde que requerem atenção e acompanhamento contínuos (danos, riscos e/ou determinantes); adoção do conceito de risco; articulação entre ações promocionais, preventivas, curativas e reabilitadoras; atuação intersetorial; ação sobre o território; e intervenção sob a forma de operações ${ }^{(16)}$.

$\mathrm{Na}$ assistência à criança na rede básica de saúde, a Agenda de Compromissos para a Saúde Integral da Criança e Redução de Mortalidade Infantil ${ }^{(2)}$ apresenta a vigilância à saúde como a postura ativa que o serviço de saúde deve assumir em situações de maior risco e dirigida a pessoas com maior vulnerabilidade, desencadeando ações estratégicas específicas para minimizar os danos com o adequado acompanhamento de saúde, programando visitas domiciliares para captação dos usuários e realização de busca ativa daqueles sem o acompanhamento programado.

Seguindo essa reflexão, destacamos o 
Novaczyk AB, Dias NS, Gaíva MAM. Atenção à saúde da criança na rede básica: análise de dissertações e teses de enfermagem. Rev. Eletr. Enf. [Internet]. 2008;10(4):1124-37. Available from: http://www.fen.ufg. br/revista/v10/n4/v10n4a25.htm.

estudo $^{(17)}$ que analisou a assistência prestada à criança de 5 a 10 anos pela equipe de saúde da família em Juiz de Fora, destacando que a assistência de saúde à criança nesta faixa etária pela equipe não está ocorrendo de forma sistematizada no conjunto das ações dos profissionais, restringindo seu atendimento de forma esporádica e pela demanda espontânea.

È essencial que a enfermeira, inserida no processo de trabalho do PSF, tome consciência da necessidade de mudança do modelo assistencial, buscando priorizar ações enfocadas na prevenção e promoção da saúde na assistência à saúde da criança, incorporando-as como instrumento na produção em saúde.

Muitos trabalhos evidenciaram que o PSF, sendo uma proposta considerada substitutiva das práticas convencionais e abrangendo dimensões técnica, política e administrativa inovadoras, requer o desenvolvimento de novas habilidades e mudanças de atitudes por parte dos profissionais.

O PSF, no processo de trabalho instrumentalizado pelo saber e tecnologia simplificados, conduz a prática de enfermagem a uma superação do senso comum do modelo assistencial curativo, embora o antagonismo desses modelos favoreça o aparecimento de muitos desencontros (T.2).

$\mathrm{Na}$ remodelação da atenção à saúde, o acolhimento é definido como uma estratégia que a equipe do PSF deve utilizar para estabelecer vínculo, reconhecer demandas e necessidades e resolver as situações que se apresentam (D.3).

(...) o trabalho do PSF vem favorecendo ações de promoção da saúde e prevenção de doenças prevalentes, com diminuição de mortes no primeiro ano de vida e efetivação do acompanhamento da saúde da criança e da família (D.11).

(...) a influência do contexto, como da organização do serviço, no modelo com PSF, promovem condições objetivas facilitadoras de uma prática mais integral (...) em relação aos profissionais de enfermagem, é preciso contextualizar que o exercício da integralidade prevê um perfil profissional com conhecimentos, habilidades e atitudes direcionados para as diretrizes da atenção básica, o que ainda representa uma grande dificuldade (T.3).

Para efetivar-se uma mudança do modelo assistencial, através do PSF, por exemplo, é necessário a implementação de mudanças no processo de trabalho em saúde, tanto no que se refere a seus propósitos ou finalidades, quanto, nos seus elementos estruturais, isto é, no objeto de trabalho, nos meios de trabalho e principalmente, nas relações estabelecidas entre eles e a população usuária dos serviços ${ }^{(18)}$.

Evidenciou-se nos trabalhos estudados, que o PSF, mesmo com todas as dificuldades, vem promovendo algumas mudanças no modelo assistencial, como por exemplo: atuação em uma base territorial, com uma clientela adscrita; atendimento humanizado, contínuo, em tempo integral; melhoria no registro das informações. As práticas dos trabalhadores de saúde e o SUS bem operacionalizado poderão ser caminhos para a desejada transformação ${ }^{(14)}$.

\section{A Enfermagem e a busca pela Assistência I ntegral à criança}

Nesta temática, buscamos relacionar as práticas de saúde na atenção à criança e a contribuição da enfermagem na busca de uma assistência integral.

A enfermagem é uma profissão que exerce diversas funções e responsabilidades no cuidado com a saúde. O cuidado ao ser humano, individualmente, na família ou na comunidade, através de atividades de promoção, prevenção, recuperação, reabilitação à saúde e trabalho em equipe, são algumas das características que definem a enfermagem, mas tem também uma dimensão educacional, para a promoção da auto-suficiência em saúde ${ }^{(11)}$.

Ao analisarmos as políticas de saúde e programas de assistência à saúde da criança, preconizadas por órgãos oficiais, percebemos a importância do profissional enfermeiro no processo de acompanhamento do crescimento e desenvolvimento infantil.

Diante disto, o enfermeiro que assiste a criança deve enfocar seu cuidado não somente na doença, no biológico, mas perceber que a criança é um ser social em desenvolvimento, que necessita ser atendida conforme sua situação social, cultural, psicológica, econômica, etc. Alguns trabalhos evidenciaram esta 
Novaczyk AB, Dias NS, Gaíva MAM. Atenção à saúde da criança na rede básica: análise de dissertações e teses de enfermagem. Rev. Eletr. Enf. [Internet]. 2008;10(4):1124-37. Available from: http://www.fen.ufg. br/revista/v10/n4/v10n4a25.htm.

abordagem:

(...) a dimensão histórico- social do cuidado à criança abriga questões sociais, culturais, políticas e econômicas agregadas às questões biológicas (D.2).

(...) a necessidade da enfermeira reconhecer as crenças que acompanham cada prática de cuidado materno ajustando às crenças, valores e modo de vida das pessoas (D.1).

A sistematização da consulta de enfermagem e a identificação dos diagnósticos de enfermagem têm contribuído para uma maior eficácia das ações do enfermeiro focalizando as intervenções necessárias para cada paciente, nas suas necessidades e dificuldades, na sua compreensão da doença, no contexto familiar e comunitário, e não somente na doença (D.10).

Estes trechos nos mostram que devemos desenvolver nossa prática de acordo com o contexto sócio-econômico-cultural de cada criança e família. O cuidado da criança e a preocupação em levá-la à idade adulta de forma saudável continuam sendo responsabilidade da família, em nossa cultura, mais especificamente da mãe, porém quando a unidade básica de saúde é procurada por esta mãe, ela necessita de orientações que condizem com seu entendimento e o contexto no qual vive. Entra neste momento, o papel de educador da enfermeira, o qual permeia todas suas atividades. E este "educar" deve ser adaptado ao tipo de clientela, ao seu contexto social e cultural.

Para desenvolver ações de educação em saúde voltadas para a população infantil é preciso reconhecer primeiramente os fatores determinantes que levam a criança a adoecer e/ou a tornar-se mais vulnerável. Para isto se faz necessário conhecer o contexto de vida dessa população(19).

No processo de educação em saúde, as informações não devem ser repassadas pelo enfermeiro sem considerar as subjetividades, as necessidades, os conhecimentos e o contexto no qual está inserida a criança e sua família. A educação em saúde deve ser um momento de trocas, de diálogo e de compartilhamento de informações. Para que as ações de educação se concretizem, as informações/saberes populares devem ser identificadas e, com a atuação da equipe de saúde, reforçadas, lapidadas e adaptadas em prol de uma melhor qualidade de $\operatorname{vida}^{(19)}$

O enfermeiro, enquanto orientador, educador, deve proporcionar às mães conhecimento satisfatório, para que estas possam desenvolver o seu cuidado com a criança da melhor forma possível. Alguns trabalhos nos mostram a importância desta função da enfermeira:

(...) os cuidados de enfermagem incluem orientar e incentivar para que haja uma maior participação materna e assim aumentar a capacidade das mães em cuidarem de seus filhos no domicílio (D.1).

O trabalho na sala de vacinas possibilita à enfermagem ser um dos profissionais da área da saúde que mais faz contatos com a criança e família nos primeiros 15 meses de vida. E toda ação que a enfermeira desenvolve tem fim pedagógico, humano (D.5).

A tese ${ }^{(14)}$ ao analisar as representações sociais da prática de enfermagem no interior dos programas de atenção à saúde da criança em UBS, identificou como uma das categorias empíricas, "enfermeira orienta", que:

(...) representa a prática de enfermagem na atenção à saúde da criança como um instrumento de ação verbal que emitem vários sentidos como: informar, educar e aconselhar as mães na prevenção de doenças mais freqüentes (T.1).

As ações de educação em saúde são inerentes ao processo de trabalho das equipes de saúde da atenção básica, que prestam atendimento à criança e com a intenção de produzir promoção e prevenção da saúde infantil.

Ao buscar a qualidade de atenção à saúde da criança é essencial o envolvimento dos profissionais, dos usuários e dos gestores para além do contexto meramente clínico, e proporcionar a mudança do modelo de atenção à saúde, principalmente na busca do sentido da integralidade.

(...) a enfermagem deve conscientizar-se do seu papel transformador, porque a interação com o outro, a intersubjetividade, o diálogo são essenciais para a busca da qualidade de vida, do autocuidado, da cidadania (D.9). 
Novaczyk AB, Dias NS, Gaíva MAM. Atenção à saúde da criança na rede básica: análise de dissertações e teses de enfermagem. Rev. Eletr. Enf. [Internet]. 2008;10(4):1124-37. Available from: http://www.fen.ufg. br/revista/v10/n4/v10n4a25.htm.

(...) a enfermeira deve ter consciência da importância do seu papel, capaz de realizar transformações possíveis, executar um trabalho com qualidade, conhecer e atuar sobre as necessidades da população (D.9).

(...) acompanhar uma criança na consulta de enfermagem em puericultura, não se resume em apenas pesar e medir, mas também associar a esta um olhar holístico sobre as condições de saúde da criança e da família, bem como, acompanha o seu desenvolvimento biopsicossocial (D.10).

(...) a importância de se engajarem (enfermagem) no trabalho da vigilância à saúde como uma proposta de mudança profunda no processo de trabalho (D.8).

Promover ações mais integrais remete a maior autonomia e independência da população em relação ao seu próprio processo saúde-doença (T.3).

Para atendermos de forma mais humanizada e individualizada a mãe e a criança, e garantir a integralidade da assistência, não podemos dissociar o contexto educativo e cultural, respeitando seus modos de vida (D.7).

Cabe aos profissionais defender a integralidade como um valor a ser sustentado e defendido nas suas práticas, ou seja, um valor que se expressa na forma como os profissionais respondem aos pacientes que os procuram ${ }^{(20)}$. E ainda acrescenta o autor $^{(20)}$ que buscar compreender o conjunto de necessidades de ações e serviços de saúde que um paciente apresenta seria, assim, a marca maior do sentido de integralidade.

\section{CONSI DERAÇÕES FI NAIS}

A maioria da produção levantada foi defendida em programas de pós-graduação da região sudeste (08) e foi desenvolvida no período de 2001 a 2005. Quanto às tendências teórico-metodológicas relativas à produção analisada prevaleceram os estudos qualitativos.

Os estudos analisados apontam para a valorização de referenciais que se centram na contextualização do processo de trabalho em saúde, no qual o enfermeiro está inserido e vem se destacando como um agente importante do trabalho.

A análise possibilitou o reconhecimento de propostas de intervenção no processo saúdedoença na infância, enfatizando o crescimento e desenvolvimento da população infantil, com propostas convergentes com as proposições políticas atuais de saúde, como o PAISC, O PSF, a estratégia AIDPI e a Agenda de compromissos para a saúde integral da criança e redução da mortalidade infantil.

Foi possível perceber através dos estudos que a prática da enfermeira ainda está inserida em um contexto centrado no modelo médico curativista. No entanto, apesar do predomínio do modelo biomédico, observaram-se inúmeros esforços para mudá-lo. As ações de promoção da saúde ainda são desenvolvidas de modo tímido, sem grande ênfase.

Para se atender a criança de forma humanizada e individualizada, pautadas na promoção da saúde e na integralidade, não se pode dissociar o contexto educativo e cultural no qual se insere a mãe e a criança. É preciso entender melhor a mãe, compreendendo e respeitando seus modos de vida e assim prestar um melhor atendimento ao seu filho. Assim, a prática profissional deve estar sempre permeada de reflexões para que se possa transformá-la gerando mudanças.

Retomando a interrogação, cabe ressaltar que, as produções de enfermagem sobre atenção à criança na rede básica, de maneira geral, realizam reflexões aprofundadas sobre novas formas de assistir ao grupo, com ênfase na promoção da saúde, integralidade e nos preceitos do SUS, visto que estes elementos têm fundamentado a formação dos enfermeiros. O que se destacava na formação dos enfermeiros, alguns anos atrás, era a valorização da técnica, do procedimento, reproduzindo o modelo biologicista. Embora este modelo ainda permeie o processo de trabalho dos profissionais da área da saúde, há um investimento maior na formação destes com os valores complexos fundamentados no SUS. Acredita-se que as mudanças, principalmente nos modos de produzir saúde, iniciam na formação dos profissionais, com a inserção da importância dos princípios do SUS nas práticas assistenciais e na mudança das relações entre equipe de saúde e criança e seu responsável, permeada numa relação cidadã e humanizada. 
Novaczyk AB, Dias NS, Gaíva MAM. Atenção à saúde da criança na rede básica: análise de dissertações e teses de enfermagem. Rev. Eletr. Enf. [Internet]. 2008;10(4):1124-37. Available from: http://www.fen.ufg. br/revista/v10/n4/v10n4a25.htm.

Dessas reflexões depreendem a necessidade de preparar profissionais/enfermeiros que sejam capazes não só de executar técnicas de trabalho, mas que sejam críticos de sua prática e dotados de competência e conhecimentos que possibilitem a compreensão do trabalho em saúde, com autonomia e capacidade de resolver problemas, e principalmente comprometidos com a ética e com a transformação da realidade da saúde infantil.

\section{REFERÊNCI AS}

1. UNICEF [Internet]. Brasília: UNICEF (BR) [cited 2008 jun 08]. Situação da Infância Brasileira 2006. Crianças de até 6 anos: o direito à sobrevivência e ao desenvolvimento. Available from: http://www.unicef.org/brazil/pt/resources_10167.htm.

2. Secretaria de Atenção à Saúde; Ministério da Saúde. Agenda de Compromissos para a Saúde Integral da Criança e Redução da Mortalidade Infantil. Brasília (Brasil): Ministério da Saúde; 2004.

3. Malveira EAP, Souza IEO. O cuidado de saúde às crianças: a posição prévia a partir das políticas públicas. Texto contexto-enferm. 1998; 7(2): 244-54.

4. Centro de Documentação do Ministério da Saúde; Ministério da Saúde. Assistência Integral à Saúde da Criança: Ações básicas. Brasília (Brasil): Ministério da Saúde; 1984.

5. Ministério da Saúde. Programa da Saúde da Família. Brasília (Brasil): Ministério da Saúde; 1997.

6. Ministério da Saúde. Atenção integrada às doenças prevalentes na infância, Curso de Capacitação. Brasília (Brasil): Ministério da Saúde; 2003.

7. Ludke $M$, André MEDA. Pesquisa em Educação: abordagens qualitativas. São Paulo: EPU; 1986.

8. Gomes R. A análise de dados em pesquisa qualitativa. In: Minayo MCS. Pesquisa Social: Teoria, Método e Criatividade. Rio de Janeiro: Vozes; 1994. p. 67-80.

9. Minayo MCS. O desafio do conhecimento: pesquisa qualitativa em saúde. São Paulo/Rio de J aneiro: Hucitec/Abrasco; 2006.

10. Santos CC. Avaliação da implementação da estratégia de Atenção Integrada às Doenças Prevalentes na Infância (AIDPI) no Estado da Bahia [dissertation]. Salvador: Escola de Enfermagem/UFB; 2004. 80 p.

11. Figueiredo GLA. A enfermagem no acompanhamento do crescimento e desenvolvimento infantil em unidade básica de saúde: fragmentos e reconstruções [dissertation]. Ribeirão Preto: Escola de Enfermagem de Ribeirão Preto/USP; 2001. 182 p.

12. Almeida MCP, Mishima SM, Silva EM, Mello DF. O trabalho de enfermagem e sua articulação com o processo de trabalho em saúde coletiva rede básica de saúde. In: Almeida MCP, Rocha SMM. O trabalho de enfermagem. São Paulo: Cortez; 1997. p. 61-111.

13. Araújo D, Miranda, MCG, Brasil SL. Formação de profissionais de saúde na perspectiva da integralidade. Revista Baiana de Saúde Pública. 2007; 31(1):20-31.

14. Monteiro Al. As representações sociais da prática de enfermagem no interior dos programas de atenção à saúde da criança de uma unidade básica de saúde do município de Natal [thesis]. Ribeirão Preto: Escola de Enfermagem de Ribeirão Preto/USP; 2000. 176 $\mathrm{p}$

15. Perillo RD. Vigilância à Saúde das Crianças menores de um ano no Centro de Saúde Goiânia, Belo Horizonte: limites e desafios [dissertation]. Belo Horizonte: Escola de Enfermagem/UFMG; 2001. 153 p.

16. Monken $\mathrm{M}$, Batistella C. Vigilância em Saúde. In: Escola Politécnica de Saúde J oaquim Venâncio. Dicionário da educação profissional em saúde. Rio de Janeiro: EPSJ V; 2006. p. 299305.

17. Camargos CCRL. Atenção à saúde da criança de 5 a 10 anos pela equipe Saúde da Família em Juiz de Fora, Belo Horizonte [dissertation]. Belo Horizonte: Escola de Enfermagem/UFMG; 2002. $142 \mathrm{p}$.

18. Teixeira CF. A Mudança do Modelo de Atenção à saúde no SUS: desatando nós, criando laços. Saúde em Debate. 2003; 27(65): 257-77.

19. Novaczyk AB. Assistência à saúde integral da criança na atenção básica: uma análise da política nacional atual [dissertation]. Cuiabá: 
Novaczyk AB, Dias NS, Gaíva MAM. Atenção à saúde da criança na rede básica: análise de dissertações e teses de enfermagem. Rev. Eletr. Enf. [Internet]. 2008;10(4):1124-37. Available from: http://www.fen.ufg. br/revista/v10/n4/v10n4a25.htm.

Faculdade de Enfermagem/UFMT; 2008. 109p.

20. Mattos RA. Os Sentidos da Integralidade: algumas reflexões acerca de valores que merecem ser defendidos. In: Pinheiro R, Mattos RA. Os sentidos da integralidade na atenção e no cuidado à saúde. Rio de Janeiro: Abrasco; 2001. p. 39-64.

Artigo recebido em 17.09.07.

Aprovado para publicação em 31.12.08. 